\title{
Quantification of I-123-meta-iodobenzylguanidine Heart-to-Mediastinum Ratios: Not So Simple After All
}

\author{
Piotr J. Slomka, PhD, ${ }^{\mathrm{a}, \mathrm{b}}$ Puja K. Mehta, MD, ${ }^{\mathrm{c}}$ Guido Germano, PhD, ${ }^{\mathrm{a}, \mathrm{b}}$ and \\ Daniel S. Berman, $M^{a, b}$ \\ ${ }^{a}$ Departments of Imaging and Medicine, and Cedars-Sinai Heart Institute, Cedars-Sinai Medical \\ Center, Los Angeles, CA \\ ${ }^{\mathrm{b}}$ David Geffen School of Medicine, University of California Los Angeles, Los Angeles, CA \\ c Barbra Streisand Women's Heart Center, Cedars-Sinai Heart Institute, Los Angeles, CA
}

Received Jun 5, 2014; accepted Jun 5, 2014

doi: 10.1007/s12350-014-9943-z

\section{See related article, pp. 970-978}

\section{WHY IS IT IMPORTANT?}

Heart failure (HF) affects over 5 million people in the United States, and while survival has improved, mortality remains high, with approximately $50 \%$ survival within 5 years of diagnosis. ${ }^{1}$ The American Heart Association estimates that prevalence of HF will increase $25 \%$ by $2030 .{ }^{1}$ Patients with $\mathrm{HF}$ and reduced ejection fraction are at risk of ventricular arrhythmias and sudden cardiac death (SCD), and current guidelines on treatment of systolic heart failure recommend implantable cardioverter defibrillator (ICD) for primary prevention of SCD in a select group of patients. ${ }^{2,3}$ The decision-making regarding placement of ICD is complex because the therapy is invasive and associated with risks, is expensive, and is not suitable for every patient. A large proportion of patients $(\approx 80 \%)$ with $\mathrm{HF}$ and reduced EF never require an ICD shock, ${ }^{4}$ and in $\approx 15 \%$ of patients, inappropriate ICD therapy occurs. ${ }^{5}$ Thus, tools to help further risk stratify which HF patient is at high risk of SCD and is likely to benefit from an ICD placement would be invaluable. It has been shown that cardiac innervation is an important factor related to

Reprint requests: Piotr J. Slomka, Departments of Imaging and Medicine, and Cedars-Sinai Heart Institute, Cedars-Sinai Medical Center, Los Angeles, CA; slomkap@cshs.org

J Nucl Cardiol 2014;21:979-83.

$1071-3581 / \$ 34.00$

Copyright (C) 2014 American Society of Nuclear Cardiology. lethal arrhythmias. ${ }^{6}$ Myocardial scintigraphy performed with Iodine-123-meta-iodobenzylguanidine (MIBG) is an imaging technique developed over 3 decades ago, capable of assessing the sympathetic denervation of the heart. ${ }^{7}$ MIBG is a well-established analog of norepinephrine, and abnormal MIBG uptake indicates abnormal cardiac sympathetic activity. Several large studies have evaluated the relationship between MIBG and $\mathrm{EF}$ with respect to adverse cardiac outcomes. ${ }^{8,9}$ These studies have shown that MIBG provides incremental prognostic information over EF in predicting cardiac death. Further, results of MIBG imaging have been shown to correlate with the occurrence of appropriate ICD shocks-patients with appropriate device discharges had significantly lower MIBG uptake as measured by traditional planar techniques. ${ }^{10,11}$

\section{MIBG IMAGING}

Iodine-123 has a half-life of 13.2 hours and predominant energy emission of $159 \mathrm{keV}$ (97\%), which is an optimal imaging energy in nuclear medicine. However, iodine-123 also emits high-energy photons of more than $400 \mathrm{keV}$ (approximately 2.9\%), with main contributing photons at $529 \mathrm{keV}$. These high energy photons pass through the collimator septa resulting in multiple Compton scatter photons, which in-turn significantly increase the background noise around the main $159 \mathrm{keV}$ energy peak, blurring the image and resulting in the relative underestimation of the counts in the myocardium as compared to the outside regions. In particular, if uptake in the nearby organs such as liver is high - the effect of scatter can be very significant despite relatively small percentage of the high energy photons, with mediastinal counts increasing over $80 \%{ }^{12}$ This effect of scatter is particularly pronounced 
when myocardial uptake is decreased-thus biasing the data in case of borderline or abnormal results. Theoretically, the scatter effects could be reduced by various mathematical methods. Scatter correction has been proposed to minimize effects of iodine-123 high-energy downscatter. ${ }^{13,14}$ Nevertheless, current scatter correction methods are approximate (especially when based on planar imaging) and non-standardized. A large variability has been observed between different implementations of scatter correction methods. ${ }^{15}$

\section{CHOICE OF COLLIMATORS}

Collimator choice for the nuclear camera substantially influences the image characteristics of MIBG. An appropriate collimator needs to be selected in consideration of septal penetration and spatial resolution. ${ }^{12}$ In nuclear medicine practice, the most commonly used collimator is the low energy general purpose (LEGP) or low energy high resolution (LEHR) collimator. It has been recognized that these collimators are not optimal for MIBG imaging due to thin septa (0.16$0.3 \mathrm{~mm}$ ) as compared with medium energy (ME) collimators with approximately $1 \mathrm{~mm}$ thick septa. The increased septal thickness of ME collimators increases the image resolution and decreases scatter in the images, but also degrades the count sensitivity and spatial resolution. The image contrast of the myocardium to background has been shown to be superior for the ME collimators. ${ }^{12}$ Thus, recent guidelines on MIBG imaging from the European Council of Nuclear Cardiology recommend the use of ME collimators for MIBG imaging. ${ }^{16}$

Unfortunately, this recommendation is not usually followed in clinical practice due to several reasons. The changing of collimators adds a significant additional effort. This may be especially cumbersome when MIBG imaging is performed in time slots between regular SPECT imaging with Tc-99m. In addition, clinicians may feel that low energy collimators are adequate based on their subjective visual analysis. Moreover, nuclear cardiology laboratories are not always equipped with ME collimators. To mitigate the scatter effects when imaging with low energy collimators, scatter correction methods have been proposed $^{17}$ but these techniques have not been yet adopted in clinical care. Thus, in clinical practice today most laboratories, especially in Europe and US use LEHR collimators without any scatter correction for MIBG imaging. In a multi-center study by Verberne et al only one center used ME collimators. ${ }^{18}$ In another large multi-center study recently reported by Jacobson et al only LEHR collimators were used in 96 centers. ${ }^{8,19}$ The situation is quite different in Japan where laboratories have longer experience with MIBG imaging, and the low-medium energy collimators, which reduce scatter from iodine-123 high energy fractions, are more widely and increasingly used.

\section{QUANTIFICATION OF MIBG UPTAKE}

Planar and SPECT techniques cannot measure absolute myocardial tracer uptake and thus only relative MIBG uptake is quantified. Although SPECT has been utilized from early days, the analysis of planar images is currently the primary standard. To determine relative myocardial MIBG uptake, the average activity is measured in the heart and the mediastinum, and a simple heart to mediastinum $(H / M)$ ratio is calculated. $H / M$ ratio is typically defined as the ratio of the counts in the heart region to the $7 \times 7$ pixel in the mediastinal region (or some other variation such as a small circular region), which is visually drawn in the mid-line upper chest position to reflect the background activity. This measure has been reported to have good observer reproducibility $^{20,21}$ and has been shown not to be affected by the differences in manually placed region of interest definitions. $^{22}$

However, $H / M$ ratio methodology has not yet been standardized between various institutions and countries. $^{23}$ SPECT quantification schemes have been proposed allowing measurement of regional uptake, ${ }^{24,25}$ but are not yet widely used. Nevertheless, even if the $H /$ $M$ ratios are reproducible, they are significantly affected by scatter from nearby organs, and acquisition parameters (such as collimator used) have been shown to be significant independent contributors to the variation of semi-quantitative MIBG uptake. ${ }^{18}$ This issue was recognized as significant ${ }^{18}$ and several efforts to standardize imaging protocols have been undertaken.

In this issue of Journal of Nuclear Cardiology ${ }^{\circledR}$, Nakajima et $\mathrm{al}^{26}$ describe the results of the large Japanese multi-center (84 institutions) initiative, reporting cross-institution phantom calibrations for the measurements of the planar $H / M$ ratio by standard nuclear cameras from a variety of vendors and collimators. The phantom design, optimized to easily produce predefined $H / M$ ratios, was previously reported by the authors. ${ }^{14}$ The authors also previously reported the initial standardization efforts in ten centers, suggesting that the phantom calibration could be used to calibrate the results between the ME and low energy collimators. ${ }^{27}$ This current work presents the data from much larger group of centers and vendors and extends the idea of the $H / M$ conversion factors to homogenize the results obtained on different types of equipment. All 84 sites performed phantom acquisitions on their cameras and 
the authors compiled a database of site-specific planar $H / M$ ratios obtained for known predefined activity concentrations in the phantom.

The results are striking. As expected, there is a significant variability in the reported $H / M$ ratios between low energy and ME collimators, but there is also wide variation of $H / M$ ratios within just one collimator type. This data is illustrated in Figure 2 in their publication. ${ }^{26}$ For example for the $H / M$ reference value of 1.6 - a value used as a possible threshold in risk stratification ${ }^{8}$ - the measured $H / M$ ratios ranged between 1.54 and 2.06 (95\% lower-upper range), depending on the site when both low energy and ME collimators are included. Even if one sub-category of the LEHR collimator is considered (authors had such data from 73 scanners) the range of correction values (0.48-0.68; median 0.55$)$ for different sites would translate into the range of $H / M$ ratio of 1.50-1.75, as illustrated in Figure 1. The data obtained with LHER collimators from different vendors was used in the recent multi-center trial of MIBG. ${ }^{8}$ To put it in the clinical context, about $26 \%$ of the patients from a multicenter prognostic study of 1,322 patients with chronic heart failure ${ }^{9}$ were within this range. ${ }^{28}$ This wide variation is troubling since no corrections to account for these variations are being applied today.

Nevertheless, these results are not surprising. Collimators with the same designation from different vendors can have quite different characteristics. For example, collimators designated as LEHR by GE and
Siemens have the following differences in parameters, respectively: hole diameter 1.5 vs $1.11 \mathrm{~mm}$, hole length 35 vs $24 \mathrm{~mm}$, septal thickness 0.2 vs $0.16 \mathrm{~mm}$, count sensitivity $0.012 \%$ vs $0.015 \% .^{12}$ It is also possible for vendors to modify the specifications of the collimators without reclassifying them to a different category. In addition, camera uniformity and other technical camera characteristics may affect $H / M$ ratio. It should be noted that the phantom study represents the best possible case, and in human subjects such inter-site variations could be expected to be larger due to added physiological variability of uptake of the organs in the field-of-view, dynamic changes in the $H / M$ ratio with time and additional scatter from organs not in the field of view (not modeled by the phantom). There are also potential issues related to different types of software used for analysis and variability in defining the regions of interest for mediastinum or heart, especially in the abnormal studies.

\section{RELATION TO PREVIOUS STUDIES}

Previously, a single $H / M$ ratio threshold of 1.6 was established as the optimal cut-off indicating abnormal sympathetic activity and for prognostication, based on the 95th percentile normal limits obtained in normal controls. ${ }^{19}$ Higher threshold 1.68 was suggested by Nakata et al in a multi-center study ${ }^{23}$ using LEHR collimators. In view of the findings by Nakajima et al, ${ }^{26}$

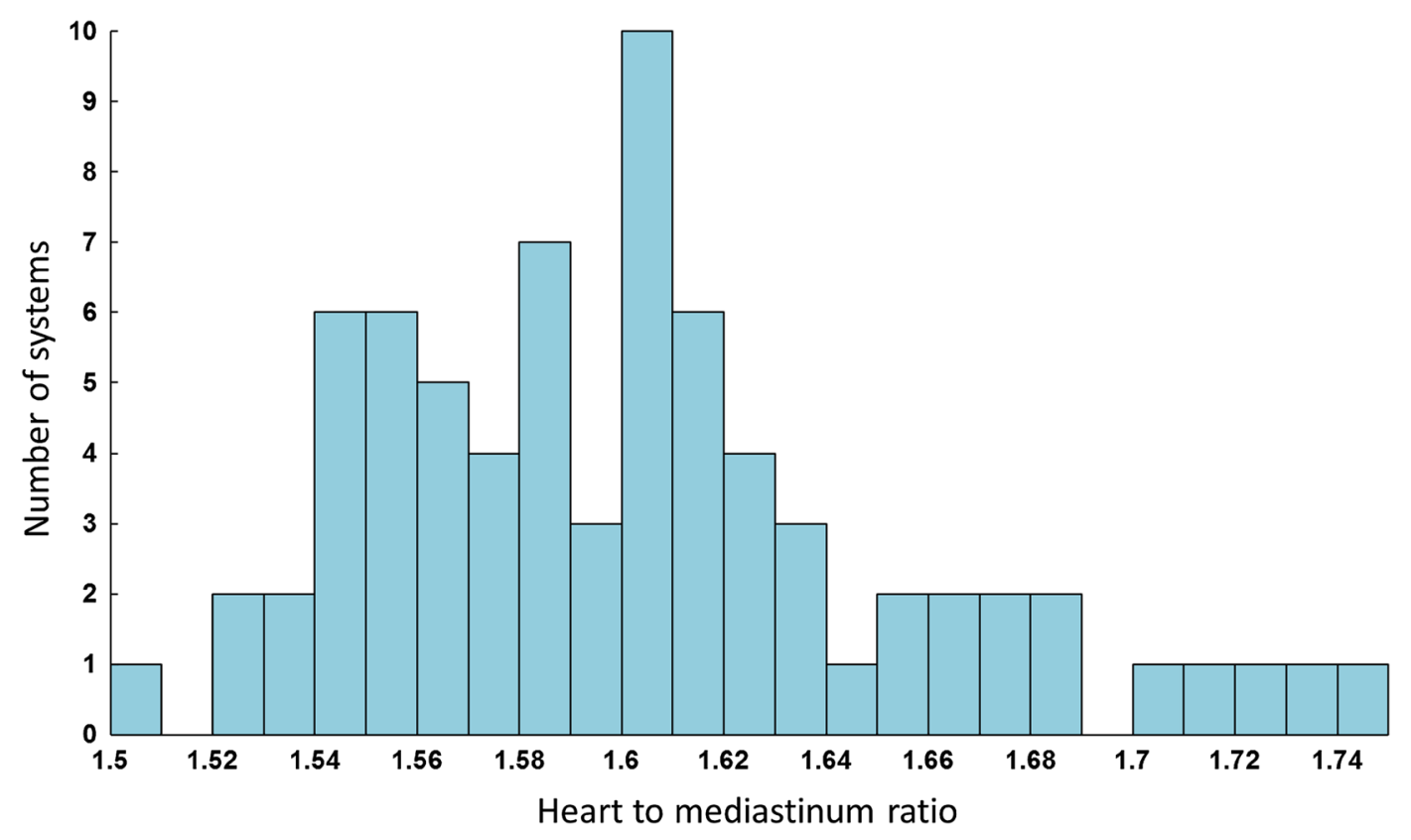

Figure 1. Simulated distribution of the heart to mediastinum $(H / M)$ ratio for the reference value of 1.6 in 73 systems with LEHR collimators, based on the phantom measurements. Five camera vendors are included. 
the use of such binary thresholds should be considered with caution. Even if there is moderate variability, it may cause misclassification of a large percentage of cases which are close to the binary threshold, resulting in a substantial gray-zone related to the test variability. ${ }^{29}$ Similar issues with the use of the dichotomous thresholds derived from the imprecise test arise in other medical applications. For example, the use of a single binary threshold of fractional flow reserve in decision of treatment for coronary artery disease has also been questioned. $^{30}$

\section{WHERE DO WE GO FROM HERE?}

The study by Nakajima et $\mathrm{al}^{26}$ addresses the variability of simple planar imaging. It is perhaps possible that better results could be obtained with quantitative SPECT imaging. New advances in SPECT software and hardware allow reconstruction of the threedimensional data with accurate attenuation and scatter correction, and depth-dependent resolution recovery modeling the characteristics of a particular collimator. ${ }^{31}$ This would potentially bring us closer to the goal of true quantitative SPECT imaging, resulting in increased accuracy of the relative uptake measures, or even in the ability to report absolute (Becquerel $/ \mathrm{ml}$ ) tracer concentration in the heart-a physicist's dream. Nevertheless, we are not there yet. Although SPECT MIBG analysis methods have been proposed, ${ }^{24,25}$ the SPECT inter-site reproducibility has not been yet shown to be superior to that of a simple planar method. Studies similar to that reported by Nakajima et al should be performed for SPECT analysis. This will be perhaps more complicated, since there are novel SPECT-only cameras now used in nuclear cardiology, with radically different collimator designs, and these devices can be used for MIBG imaging. ${ }^{32}$ These new scanners, based on solid state Cadmium-Zinc-Telluride detectors offer additional potential to improve the quantitative MIBG accuracy due to much higher photon energy resolutionand thus significantly better ability to discriminate scattered photons.

However, before we can develop and validate such highly accurate methods with the use of SPECT and the new generation of scanners, there are several immediate recommendations, which could be proposed, based on the available data for planar MIBG imaging and the study by Nakajima et al. Phantom calibration for each center performing MIBG imaging will be very beneficial and is less complicated than the development of site-specific normal limits. Perhaps if phantom measurements for the identical system configuration have been already recorded, they could be retrieved from the database rather than re- measured-but this should be confirmed first by multi-site tests. The logistics of such phantom calibration should be discussed by the international MIBG community, considering the extensive and valuable work performed in this regard by our Japanese colleagues. Even if such phantom calibration does not account for all the variability, it will significantly improve the device-independence and thus allow intercomparison of the multi-site results, or even serial test results at the same institution. The MIBG phantom calibration could even be performed retrospectively and appropriate corrections could be applied to the already acquired multi-center data. Perhaps re-analysis of the calibrated data could result in improved results from several trials conducted so far. As for the use of the single threshold for the $H / M$ ratio, it should be recognized that there is a specific range of the $H / M$ ratios, where no definite prognosis or treatment decision could be made on the basis of the MIBG scan alone, due to the inherent variability of the test. Thus, only more definitive $H / M$ ratio measurements that are outside of the gray-zone range would be used to guide therapy. This decision-making could be modeled by a continuous probabilistic analysis with outcome scores assigned to the given MIBG result, rather than guided by a single threshold.

In summary, the measurement of $H / M$ planar ratio from MIBG is deceivingly simple, but it may not be that simple after all.

\section{Acknowledgments}

Dr Mehta is supported by the National Institutes of Health Grant K23HL105787.

\section{Disclosures}

None.

\section{References}

1. Go AS, Mozaffarian D, Roger VL, Benjamin EJ, Berry JD, Blaha MJ, et al. Heart disease and stroke statistics-2014 update: A report from the American Heart Association. Circulation 2014;129:e28-292.

2. Lindenfeld J, Albert NM, Boehmer JP, Collins SP, Ezekowitz JA, Givertz MM, et al. HFSA 2010 Comprehensive Heart Failure Practice Guideline. J Card Fail 2010;16:e1-194.

3. Hunt SA, Abraham WT, Chin MH, Feldman AM, Francis GS, Ganiats TG, et al. 2009 focused update incorporated into the ACC/ AHA 2005 guidelines for the diagnosis and management of heart failure in adults: A report of the American College of Cardiology Foundation/American Heart Association Task Force on Practice Guidelines Developed in Collaboration with the International 
Society for Heart and Lung Transplantation. J Am Coll Cardiol 2009;53:e1-90.

4. Tung R, Zimetbaum P, Josephson ME. A critical appraisal of implantable cardioverter-defibrillator therapy for the prevention of sudden cardiac death. J Am Coll Cardiol 2008;52:1111-21.

5. Sweeney MO, Wathen MS, Volosin K, Abdalla I, DeGroot PJ, Otterness MF, et al. Appropriate and inappropriate ventricular therapies, quality of life, and mortality among primary and secondary prevention implantable cardioverter defibrillator patients: Results from the Pacing Fast VT REduces Shock ThErapies (PainFREE Rx II) trial. Circulation 2005;111:2898-905.

6. Podrid PJ, Fuchs T, Candinas R. Role of the sympathetic nervous system in the genesis of ventricular arrhythmia. Circulation 1990;82:I103-13.

7. Kline RC, Swanson DP, Wieland DM, Thrall JH, Gross MD, Pitt $\mathrm{B}$, et al. Myocardial imaging in man with I-123 meta-iodobenzylguanidine. J Nucl Med 1981;22:129-32.

8. Jacobson AF, Senior R, Cerqueira MD, Wong ND, Thomas GS, Lopez VA, et al. Myocardial iodine-123 meta-iodobenzylguanidine imaging and cardiac events in heart failure. Results of the prospective ADMIRE-HF (AdreView Myocardial Imaging for Risk Evaluation in Heart Failure) study. J Am Coll Cardiol 2010;55:2212-21.

9. Nakajima K, Nakata T, Yamada T, Yamashina S, Momose M, Kasama S, et al. A prediction model for 5-year cardiac mortality in patients with chronic heart failure using I-metaiodobenzylguanidine imaging. Eur J Nucl Med Mol Imaging 2014. doi: 10.1007/s00259-014-2759-x

10. Arora R, Ferrick KJ, Nakata T, Kaplan RC, Rozengarten M, Latif F, et al. I-123 MIBG imaging and heart rate variability analysis to predict the need for an implantable cardioverter defibrillator. J Nucl Cardiol 2003;10:121-31.

11. Boogers MJ, Borleffs CJ, Henneman MM, van Bommel RJ, van Ramshorst J, Boersma E, et al. Cardiac sympathetic denervation assessed with 123-iodine metaiodobenzylguanidine imaging predicts ventricular arrhythmias in implantable cardioverterdefibrillator patients. J Am Coll Cardiol 2010;55:2769-77.

12. Verberne HJ, Feenstra C, de Jong WM, Somsen GA, van Eck-Smit BL, Busemann Sokole E. Influence of collimator choice and simulated clinical conditions on 123I-MIBG heart/mediastinum ratios: A phantom study. Eur $\mathrm{J}$ Nucl Med Mol Imaging 2005:32:1100-7.

13. Small AD, Prosser J, Motherwell DW, McCurrach GM, Fletcher AM, Martin W. Downscatter correction and choice of collimator in 123I imaging. Phys Med Biol 2006;51:N307-11.

14. Nakajima $K$, Matsubara $K$, Ishikawa $T$, Motomura N, Maeda R, Akhter N, et al. Correction of iodine-123-labeled meta-iodobenzylguanidine uptake with multi-window methods for standardization of the heart-to-mediastinum ratio. J Nucl Cardiol 2007;14:843-51

15. Zaidi H, Koral KF. Scatter modelling and compensation in emission tomography. Eur J Nucl Med Mol Imaging 2004;31:761-82.

16. Flotats A, Carrio I, Agostini D, Guludec D, Marcassa C, Schäfers M, et al. Proposal for standardization of 123I-metaiodobenzylguanidine (MIBG) cardiac sympathetic imaging by the EANM Cardiovascular Committee and the European Council of Nuclear Cardiology. Eur J Nucl Med Mol Imaging 2010;37:1802-12.

17. Fletcher AM, Motherwell DW, Small AD, McCurrach GM, Goodfield NE, Petrie MC, et al. I-123 MIBG cardiac uptake measurements: Limitations of collimator choice and scatter correction in the clinical context. Nucl Med Commun 2010;31:629-36.
18. Verberne HJ, Habraken JB, van Eck-Smit BL, Agostini D, Jacobson AF. Variations in 123I-metaiodobenzylguanidine (MIBG) late heart mediastinal ratios in chronic heart failure: A need for standardisation and validation. Eur $\mathrm{J}$ Nucl Med Mol Imaging 2008;35:547-53.

19. Jacobson AF, Lombard J, Banerjee G, Camici PG. 123I-mIBG scintigraphy to predict risk for adverse cardiac outcomes in heart failure patients: Design of two prospective multicenter international trials. J Nucl Cardiol 2009;16:113-21.

20. Pellegrino T, Petretta M, De Luca S, Paolillo S, Boemio A, Carotenuto $\mathrm{R}$, et al. Observer reproducibility of results from a lowdose 123I-metaiodobenzylguanidine cardiac imaging protocol in patients with heart failure. Eur $\mathrm{J}$ Nucl Med Mol Imaging 2013;40:1549-57.

21. Veltman CE, Boogers MJ, Meinardi JE, Al Younis I, DibbetsSchneider P, Van der Wall EE, et al. Reproducibility of planar (123)I-meta-iodobenzylguanidine (MIBG) myocardial scintigraphy in patients with heart failure. Eur J Nucl Med Mol Imaging 2012;39:1599-608.

22. Jacobson AF, Matsuoka DT. Influence of myocardial region of interest definition on quantitative analysis of planar 123I-mIBG images. Eur J Nucl Med Mol Imaging 2013;40:558-64.

23. Nakata T, Nakajima K, Yamashina S, Yamada T, Momose M, Kasama S, et al. A pooled analysis of multicenter cohort studies of (123)I-mIBG imaging of sympathetic innervation for assessment of long-term prognosis in heart failure. JACC Cardiovasc Imaging 2013;6:772-84.

24. Chen J, Folks RD, Verdes L, Manatunga DN, Jacobson AF, Garcia EV. Quantitative I-123 mIBG SPECT in differentiating abnormal and normal mIBG myocardial uptake. J Nucl Cardiol 2012;19:92-9.

25. van der Veen BJ, Al Younis I, de Roos A, Stokkel MP. Assessment of global cardiac I-123 MIBG uptake and washout using volumetric quantification of SPECT acquisitions. J Nucl Cardiol 2012;19:752-62.

26. Nakajima K, Okuda K, Yoshimura M, Matsuo S, Wakabayashi H, Imanishi $\mathrm{Y}$, et al. Multicenter cross-calibration of I-123 metaiodobenzylguanidine heart-to-mediastinum ratios to overcome camera-collimator variations. J Nucl Cardiol 2014. doi:10.1007/ s12350-014-9916-2

27. Nakajima K, Okuda K, Matsuo S, Yoshita M, Taki J, Yamada M, et al. Standardization of metaiodobenzylguanidine heart to mediastinum ratio using a calibration phantom: Effects of correction on normal databases and a multicentre study. Eur J Nucl Med Mol Imaging 2012;39:113-9.

28. Nakajima K. Distributution of $\mathrm{H} / \mathrm{M}$ ratios in the multicenter trial of MIBG. (personal communication) 2014.

29. Petretta M, Pellegrino T, Cuocolo A. The "gray zone" for the heart to mediastinum MIBG uptake ratio. J Nucl Cardiol 2014. doi:10.1007/s12350-014-9894-4

30. Lindstaedt M, Yazar A, Germing A, Fritz MK, Holland-Letz T, Mügge A, et al. Clinical outcome in patients with intermediate or equivocal left main coronary artery disease after deferral of surgical revascularization on the basis of fractional flow reserve measurements. Am Heart J 2006;152:156 e151-9.

31. Slomka PJ, Patton JA, Berman DS, Germano G. Advances in technical aspects of myocardial perfusion SPECT imaging. J Nucl Cardiol 2009;16:255-76

32. Ben-Haim S, Menichetti F, Allie R, Roth N, Baavour R, Rubens $M$, et al. Simultaneous dual-radionuclide imaging with 99mTechnetium-MIBI and 123I-mIBG in patients with ventricular arrhythmia_-Initial experience. J Nucl Med. 2014;55:1723. 